\title{
THE LIFETIME OF A RANDOM SET
}

\author{
PETER C. KIESSLER *** AND \\ KANOKTIP NIMITKIATKLAI, ${ }^{*}$ Clemson University
}

\begin{abstract}
We consider the lifetimes of systems that can be modeled as particles that move within a bounded region in $\mathbb{R}^{n}$. Particles move within the set according to a random walk, and particles that leave the set are lost. We divide the set into equal cells and define the lifetime of the set as the time required for the number of particles in one of the cells to fall below a predetermined threshold. We show that the lifetime of the system, given a sufficiently large number of particles, is Weibull distributed.
\end{abstract}

Keywords: Weibull distribution; extreme value theory; interacting particle system

2000 Mathematics Subject Classification: Primary 60G70

Secondary 90B25

\section{Introduction}

We consider a sequence $\left\{X^{(N)}\right\}$ of particle systems. In $X^{(N)}$, for a given positive integer $N$, particles move on the $d$-dimensional lattice $\{1, \ldots, l N\}^{d}$, each according to a continuous-time random walk. A particle stays at a lattice point for an exponential amount of time having mean $u_{N}^{-1}$. Upon leaving the lattice point, the particle is equally likely to visit any of the $2 d$ adjacent lattice points in $\mathbb{Z}_{+}^{d}$. If the particle moves to a point outside the lattice $\{1, \ldots, l N\}^{d}$, it dies. We divide the lattice into $N^{d}$ cells, each being a cube of $l^{d}$ vertices. Initially, each cell contains $\mathcal{K}$ particles, and the initial configuration of particles within a cell is random. The lifetime $\tau_{N}$ of $X^{(N)}$ is defined to be the amount of time required for the number of particles in at least one cell to fall below $\mathcal{K}-\kappa$. We will show that $\tau_{N}$ converges in distribution to a Weibull-distributed random variable.

The lifetime of $X^{(N)}$ is the minimum of the lifetimes of the $N^{d}$ cells. Since the cells are homogeneous, in the sense that each starts with a random initial configuration of particles and that each particle moves according to the same law, one suspects that the limit distribution, provided that it exists, is an extreme value distribution. Since the lifetime of a cell is a first passage time in a Markov process having a discrete state space, it has a distribution that is an 'infinite' mixture of gamma distributions, and is thus in the domain of attraction of a Weibull distribution. That particles move between cells causes the lifetimes of the cells to be dependent random variables, and the boundary causes the lifetimes not to be identically distributed. The lifetime distributions of the cells are shown to be regularly varying at 0 with an index that does not depend on the cell. Bingham et al. [2] and Resnick [9] are excellent references to regularly varying functions and their application to extreme value theory. To account for the dependencies between the cells, we show that the lifetimes of the cells satisfy modified versions of the mixing conditions $D\left(u_{N}\right)$ and $D^{\prime}\left(u_{N}\right)$ given in [5].

Received 23 August 2004; revision received 12 November 2004.

* Postal address: Department of Mathematical Sciences, Clemson University, Clemson, SC 29634-0975, USA.

** Email address: kiesslp@ces.clemson.edu 
Our interest in the problem stems from considerations in reliability theory. As an extreme value distribution, the Weibull distribution arises as a normalized limit of the minimum of independent and identically distributed random variables. For this reason, it is often used in reliability applications as a lifetime distribution. Gumbel [4] and Barlow and Proschan [1] provided several examples of how the Weibull distribution appears in reliability applications as a limit of the minimum of independent random variables. Lawless [6] and Meeker and Escobar [7] are more recent references, which cite the role of extreme value distributions in reliability and, hence, the appearance of the Weibull distribution in many reliability studies.

Our model can be used to determine the time until a defect occurs in a heterogeneous material. Initially, the material has a uniform structure with no defects. As time evolves, the material develops imperfections or defects. The presence of defects is undesirable in materials such as silicon wafers, as they can affect various aspects of semiconductor manufacturing, from production yields to product reliability; see [8] and [3]. We consider a defect to occur when the amount of material in some cell falls below a predetermined threshold. Our result states that the time until a defect occurs is approximately Weibull distributed.

In Section 2, we state the problem and our results. In particular, we model the movement of particles in the set as a particle system that is a Markov process on a finite state space. The lifetime of the system is then defined as the amount of time it takes for the Markov process to reach a given subset of the state space. Due to the size of the state space, calculating the lifetime distribution as a passage problem will be quite formidable and, hence, we seek to approximate the lifetime distribution using extreme value theory. We then state Theorem 2.1 and Corollary 2.1, and complete Section 2 by motivating the proof of Theorem 2.1, which is given in Section 3.

\section{Problem description}

We construct a sequence of particle systems, each of which is a Markov process on a finite state space. The lifetime of a particle system is formulated as a passage time in the Markov process. Calculating the passage time distribution explicitly appears to be a formidable problem, so we seek a limit theorem. Theorem 2.1 and Corollary 2.1 give conditions under which the lifetime distributions converge to a Weibull distribution.

For $N=1,2, \ldots$, we consider the graph $\left(S_{N}, E_{N}\right)$. The vertices $S_{N}$ are those of the cube $\{1, \ldots, l N\}^{d} \subset \mathbb{Z}^{d}$. There is an edge connecting $\bar{\imath}=\left(i_{1}, \ldots, i_{d}\right)$ and $\bar{j}=\left(j_{1}, \ldots, j_{d}\right)$ if $\sum_{k=1}^{d}\left|i_{k}-j_{k}\right|=1$. Divide $S_{N}$ into $N^{d}$ equal cubes, which we call cells. Each cell is a cube containing $l^{d}$ vertices. We regard each vertex as a location where particles reside and there are $(l N)^{d}$ such locations. Initially, there are $\mathcal{P}_{N}$ particles in the set. Each particle evolves according to a random walk, and once the particle leaves $S_{N}$ it dies. The process that describes the superposition of these random walks is the continuous-time Markov chain that gives the number of particles at each vertex of the set.

To account for particles that die, we append to each $S_{N}$ a cemetery point $\Delta$, not contained in $S_{N}$, and set $S_{N, \Delta}=S_{N} \cup\{\Delta\} . S_{N, \Delta}$ will denote the state space for the Markov process that describes the movement of a single particle. To keep track of the movement of particles, we introduce the mappings $T^{k}$ and $T_{k}, k=1, \ldots, d$, from $S_{N}$ to $\mathbb{Z}^{d}$, which are defined by

$$
\left(T^{k}(\bar{\imath})\right)_{j}= \begin{cases}i_{j} & \text { if } j \neq k \\ i_{k}+1 & \text { if } j=k\end{cases}
$$


and

$$
\left(T_{k}(\bar{\imath})\right)_{j}= \begin{cases}i_{j} & \text { if } j \neq k \\ i_{k}-1 & \text { if } j=k\end{cases}
$$

Let $\Delta(\bar{l})$ be those $T^{k}(\bar{l})$ and $T_{k}(\bar{l})$ that do not belong to $S_{N} \subset \mathbb{Z}^{d}$ and let $|\Delta(\bar{l})|$ be the number of points in $\Delta(\bar{l})$. We model the movement of a particle as the Markov process, on the state space $S_{N, \Delta}$, whose generator is given by

$$
a_{\bar{l}, \bar{\jmath}}= \begin{cases}\frac{\lambda}{2^{d}} & \text { if } \bar{j} \in \bigcup_{k=1}^{d}\left\{T^{k}(\bar{\imath}), T_{k}(\bar{\imath})\right\} \cap S_{N}, \\ \frac{|\Delta(\bar{l})| \lambda}{2^{d}} & \text { if } \bar{\jmath}=\Delta, \\ -\lambda & \text { if } \bar{j}=\bar{l}, \\ 0 & \text { otherwise }\end{cases}
$$

where $\bar{l} \in S_{N}$ and $\bar{j} \in S_{N, \Delta}$. Let $P_{t}$ denote the transition function of the Markov process. We remind the reader that

$$
P_{t}(\Delta, \bar{\jmath})= \begin{cases}1 & \text { if } \bar{j}=\Delta \\ 0 & \text { otherwise }\end{cases}
$$

The particle system $X^{(N)}$ gives the number of particles at each location. The state space $\mathcal{S}_{N}$ of $X^{(N)}$ is the set of all vectors

$$
\left\{s_{\bar{\jmath}}: \bar{j} \in S_{N, \Delta}, s_{\bar{j}} \geq 0, \sum_{\bar{j} \in S_{N, \Delta}} s_{\bar{j}}=\mathcal{P}_{N}\right\} .
$$

We assume that the particles move independently and that any number of them can occupy a given location. The above assumptions imply that $X^{(N)}$ is a Markov process.

The construction is such that the process describing the movement of a single particle can be formulated as a uniformized Markov process by allowing a dead particle to jump from $\Delta$ to $\Delta$. Thus, the jump times of individual particles form a Poisson process with constant rate $\lambda$. Since the particles move independently, the jump times corresponding to a subset of the set of particles will form a Poisson process with rate equal to $\lambda$ times the number of particles in the subset. We will exploit this property often in the proof of the theorem.

Initially, the cell configurations are independent and identically distributed. Each starts with $\mathcal{K}$ particles, whose initial locations are chosen according to an arbitrary distribution, and lives until the number of particles it contains falls below $\mathcal{K}-\kappa$. Recall that the lifetime of $\mathcal{X}^{(N)}$ is the minimum of the lifetimes of the cells. Let $\mathcal{B}_{N}$ be the subset of $\mathcal{S}_{N}$ that consists of those states for which at least one cell has fewer than $\mathcal{K}-\kappa$ particles. The lifetime of $\mathcal{X}^{(N)}$ is then the length of time it takes the process to enter $\mathcal{B}_{N}$. For large $N$, the set $\mathcal{B}_{N}$ is complicated, which makes explicit calculation of the lifetime distribution quite formidable. Our main result is that, for large $N$, the distribution of the lifetime of $X^{(N)}$ is approximately Weibull.

To state the result, consider the possible initial configurations of the particles in a cell; there are $\mathcal{K}^{l^{d}}$ such configurations. For the $h$ th configuration, let $x_{(k)}^{h}$ be the minimum number of 
transitions required for the $k$ th particle to leave the cell. We number the particles in the cell such that $x_{(1)}^{h} \leq \cdots \leq x_{(\mathcal{K})}^{h}$, and we let

$$
n_{h}=\sum_{k=1}^{\kappa} x_{(k)}^{h}
$$

In addition, we let $q_{h}$ be the probability that the $h$ th configuration is chosen and let $c_{h}$ be the probability that $n_{h}$ jumps in the cell result in $\kappa$ particles leaving the cell. Finally, we set

$$
n_{0}=\min \left\{n_{h}: q_{h}>0\right\} \quad \text { and } \quad H=\left\{h: n_{h}=n_{0}\right\},
$$

and we let

$$
q=\sum_{h \in H} q_{h} c_{h} \quad \text { and } \quad u_{N}=\frac{1}{\mathcal{K}}\left(\frac{n_{0} !}{N^{d}}\right)^{1 / n_{0}} .
$$

Theorem 2.1. Let $\tau_{N}$ denote the lifetime of $X^{(N)}$, with $\lambda=1$. Then, with $n_{0}$ and $u_{N}$ as defined above,

$$
\lim _{N \rightarrow \infty} \mathrm{P}\left\{\tau_{N}>x u_{N}\right\}=\mathrm{e}^{-q x^{n} 0}
$$

for all $x>0$.

The following corollary is perhaps more useful in applications.

Corollary 2.1. Let the rate at which particles move in the Nth system be $u_{N}$. Then, with the notation of Theorem 2.1,

$$
\lim _{N \rightarrow \infty} \mathrm{P}\left\{\tau_{N}>x\right\}=\mathrm{e}^{-q x^{n} 0}
$$

for all $x>0$.

The proof of Theorem 2.1 is given in Section 3. It is long but it is motivated by some basic ideas from extreme value theory, which we now recall. Suppose that we are given independent and identically distributed random variables having common distribution $F$. To show that there exist $u_{N}$ with $\lim _{N \rightarrow \infty} u_{N}=0$, such that

$$
\lim _{N \rightarrow \infty}\left(1-F\left(u_{N} x\right)\right)^{N^{d}}=\mathrm{e}^{-x^{n_{0}}},
$$

it suffices to show that

$$
\lim _{N \rightarrow \infty} N^{d} F\left(u_{N}\right)=1
$$

and that

$$
\lim _{N \rightarrow \infty} \frac{F\left(u_{N} x\right)}{F\left(u_{N}\right)}=x^{n_{0}} .
$$

To see this, substitute (2.3) into (2.2) to obtain

$$
\lim _{N \rightarrow \infty} N^{d} F\left(u_{N} x\right)=x^{n_{0}},
$$

which implies (2.1).

If the random variables are stationary, under suitable mixing conditions (2.2) and (2.3) still imply (2.1), as was shown in [5]. We provide a brief outline of the approach taken in [5]. Suppose that $\left\{X_{\bar{n}}, \bar{n} \in N^{d}\right\}$ form a stationary sequence of random variables, and again let $F$ 
denote their common distribution. The approach in [5] is to show, under appropriate conditions, that (2.2) implies

$$
\lim _{N \rightarrow \infty} \mathrm{P}\left\{\min _{\bar{n} \in B_{N}} X_{\bar{n}}>u_{N}\right\}=\mathrm{e}^{-1},
$$

where $B_{N}=\{1, \ldots, N\}^{d}$. Combining (2.4) with (2.3) further shows that

$$
\lim _{N \rightarrow \infty} \mathrm{P}\left\{\min _{\bar{n} \in B_{N}} X_{\bar{n}}>u_{N} x\right\}=\mathrm{e}^{-x^{n_{0}}} .
$$

In [5], it is shown that (2.2) implies (2.4) in two steps. First, if the mixing condition $D\left(u_{N}\right)$ (of [5]) holds then, for any positive integer $k$,

$$
\lim _{N \rightarrow \infty}\left(\mathrm{P}\left\{\min _{\bar{n} \in B_{N}} X_{\bar{n}}>u_{N}\right\}-P^{k^{d}}\left\{\min _{\bar{n} \in B_{[N / k]}} X_{\bar{n}}>u_{N}\right\}\right)=0 .
$$

Second, the condition $D^{\prime}\left(u_{N}\right)$ (of [5]), together with (2.2), implies that

$$
\begin{aligned}
1-k^{-d} & \leq \liminf _{N \rightarrow \infty} \mathrm{P}\left\{\min _{\bar{n} \in B_{[N / k]}} X_{\bar{n}}>u_{N}\right\} \\
& \leq \limsup _{N \rightarrow \infty} \mathrm{P}\left\{\min _{\bar{n} \in B_{[N / k]}} X_{\bar{n}}>u_{N}\right\} \\
& \leq\left(1-k^{-d}+o\left(k^{-d}\right)\right) .
\end{aligned}
$$

Taking the $k^{d}$ th power of each term, using (2.5), and then letting $k \rightarrow \infty$ gives (2.4) and, hence, the result.

In our setting, the lifetime distributions of distinct cells are usually different. Our arguments show that the differences between the lifetime distributions are small enough that the approach suggested in [5] still works. To apply this approach, we must identify the $u_{N}$, give analogs of (2.2) and (2.3), develop minor modifications to $D\left(u_{N}\right)$ and $D^{\prime}\left(u_{N}\right)$, and show that these conditions imply our versions of (2.5) and (2.6). This is done as follows: Lemmas 3.2 and 3.3 identify the $u_{N}$ and give the conditions analogous to (2.2) and (2.3), Lemmas 3.4 and 3.5 develop our analog of condition $D\left(u_{N}\right)$, Lemmas 3.6, 3.7, and 3.8 give our version of (2.5), Lemma 3.9 gives the condition $D^{\prime}\left(u_{N}\right)$, and Lemma 3.10 shows that (2.6) holds.

\section{Proof of Theorem 2.1}

Recall that $S_{N}=\{1, \ldots, l N\}^{d}$ consists of $(l N)^{d}$ locations (vertices) and $N^{d}$ cells. For $\bar{J}=$ $\left(j_{1}, \ldots, j_{d}\right), j_{i}=1, \ldots, N$, let $C_{N, j}$ be the cell consisting of the locations $\prod_{i=1}^{d}\left\{l\left(j_{i}-1\right)+1\right.$, $\left.\ldots, l j_{i}\right\}$ and let $\tau_{N, \bar{j}}$ denote its lifetime. For each $N, \tau_{N}=\min _{\bar{j}} \tau_{N, \bar{j}}$.

We will show that there exists a sequence $u_{N} \rightarrow 0$ such that

$$
\begin{aligned}
\lim _{N \rightarrow \infty} \mathrm{P}\left\{\tau_{N}>x u_{N}\right\} & =\lim _{N \rightarrow \infty} \mathrm{P}\left\{\min _{\bar{j}} \tau_{N, \bar{j}}>x u_{N}\right\} \\
& =\mathrm{e}^{-q x^{n} 0}
\end{aligned}
$$

for all $x>0$. Since $\tau_{N, \bar{j}}$ is a first passage time in a Markov process, its distribution is absolutely continuous and has support $[0, \infty)$. Thus, there is a $u_{N}$ such that

$$
\mathrm{P}\left\{\tau_{N, \bar{j}} \leq u_{N}\right\}=\frac{q}{N^{d}} .
$$


This $u_{N}$ depends not only on $N$, but also on the location of the cell $C_{N, \bar{j}}$ and, hence, on $\bar{j}$. Below, we will show how to determine a $u_{N}$ such that

$$
\mathrm{P}\left\{\tau_{N, \bar{j}} \leq u_{N}\right\}=\frac{q}{N^{d}}+o\left(\frac{1}{N^{d}}\right),
$$

with $u_{N}$ independent of $\bar{j}$. We determine $u_{N}$ by comparing $\tau_{N, \bar{j}}$ to $\tau_{1, \overline{1}}$, the lifetime of a system consisting of the cell $C_{1, \overline{1}}$, which consists of the locations $\{1, \ldots, l\}^{d}$, and to the lifetime of a cell in an infinite system, which we now construct.

Consider a system on the lattice $\mathbb{Z}^{d}$. Cells consist of the points $\left\{l\left(k_{1}-1\right)+1, \ldots, l k_{1}\right\} \times$ $\cdots \times\left\{l\left(k_{d}-1\right)+1, \ldots, l k_{d}\right\}$, where $\left(k_{1}, \ldots, k_{d}\right) \in \mathbb{Z}^{d}$. The particles move independently of each other and each according to a random walk, as in $S_{N}$. However, there is no boundary and so there is no cemetery point $\Delta$. Initially, all cells are independent and identically distributed and have the same distribution as those of $X^{(N)}$. We refer to this system as the infinite system.

Let $\tilde{\tau}_{\overline{1}}$ be the lifetime of the cell $\tilde{C}_{\overline{1}}$ consisting of the locations $\{1, \ldots, l\}^{d}$ in the infinite system. The following coupling argument shows that $\tau_{1, \overline{1}}$ is stochastically smaller than $\tau_{N, \bar{j}}$, which is stochastically smaller than $\tilde{\tau}_{\overline{1}}$. Note that $\tau_{1, \overline{1}}$ is the lifetime of $S_{1}$, which consists of the single cell $C_{1, \overline{1}}$.

Let $C_{N, \bar{j}}$ be a cell in system $S_{N}$. Using the fact that $C_{N, \bar{j}} \subset S_{N} \subset S_{\infty}=\mathbb{Z}^{d}$, we color white the particles that start in $C_{N, \bar{j}}$, color orange those that start in $S_{N} / C_{N, \bar{j}}$, and color the remainder purple. Let the particles move as in the infinite system. When a white particle leaves $C_{N, \bar{j}}$, it changes color to orange if it enters $S_{N}$, and to purple otherwise. When an orange particle leaves $S_{N}$, it changes color to purple. We will say that $C_{N, \bar{j}}$ dies in $\tilde{X}^{(1)}$ (the process giving the locations of the white particles) when the number of white particles in $C_{N, \bar{j}}$ falls below $\mathcal{K}-\kappa$, that $C_{N, \bar{j}}$ dies in $\mathcal{X}^{(N)}$ when the combined number of white and orange particles in $C_{N, \bar{j}}$ falls below $\mathcal{K}-\kappa$, and that $C_{N, \bar{j}}$ dies in the infinite system when the number of particles (of any color) in $C_{N, \bar{j}}$ falls below $\mathcal{K}-\kappa$. Since $\tilde{X}^{(1)}$ only tracks white particles, the lifetime of $C_{N, \bar{j}}$ in $\tilde{X}^{(1)}$ has the same distribution as $\tau_{1, \overline{1}}$. Moreover, the lifetime of $C_{N, \bar{j}}$ in $X^{(N)}$ has the same distribution as $\tau_{N, \bar{j}}$. In the infinite system, all cells have the same lifetime distribution and, hence, the lifetime of $C_{N, \bar{j}}$ in the infinite system is $\tilde{\tau}_{\overline{1}}$. It is clear that the lifetime of $C_{N, \bar{j}}$ is smallest in $\tilde{X}^{(1)}$ and largest in the infinite system. The result now follows.

For each cell $C_{N, \bar{j}} \in S_{N}$, the movement of the $\mathcal{K}$ particles originally belonging to $C_{N, \bar{j}}$ can be described as follows. Let $\left\{T_{n}^{(N, \bar{j})}, n=1,2, \ldots\right\}$ be the jump times of a Poisson process of rate $\mathcal{K}$. At the $n$th jump time, randomly choose one of the $\mathcal{K}$ particles that originally belonged to $C_{N, \bar{j}}$. If the chosen particle is in $\Delta$ it stays there; otherwise, it is equally likely to move in any of the $2 d$ directions to a new location, possibly $\Delta$. Let $\left\{X_{n}^{(N, \bar{j})}, n=0,1, \ldots\right\}$ be the Markov chain in which $X_{n}^{(N, \bar{J})}$ gives the locations of the $\mathcal{K}$ particles at time $T_{n}^{(N, \bar{\jmath})}$. Then

$$
\begin{aligned}
\mathrm{P}\left\{\tau_{N, \bar{j}} \leq t\right\}= & \sum_{n=n_{0}}^{\infty} \mathrm{P}\left\{\tau_{N, \bar{j}} \leq t, T_{n}^{(N, \bar{j})} \leq t<T_{n+1}^{(N, \bar{j})}\right\} \\
= & \mathrm{P}\left\{\tau_{N, \bar{j}} \leq t, T_{n_{0}}^{(N, \bar{\jmath})} \leq t<T_{n_{0}+1}^{(N, \bar{j})}\right\} \\
& +\sum_{n=n_{0}+1}^{\infty} \mathrm{P}\left\{\tau_{N, \bar{j}} \leq t, T_{n}^{(N, \bar{j})} \leq t<T_{n+1}^{(N, \bar{j})}\right\} .
\end{aligned}
$$

The term on the last line of (3.1) is bounded above by

$$
\sum_{n=n_{0}+1}^{\infty} \frac{(\mathcal{K} t)^{n}}{n !} \mathrm{e}^{-\mathcal{K} t} \leq \frac{(\mathcal{K} t)^{n_{0}+1}}{\left(n_{0}+1\right) !}
$$




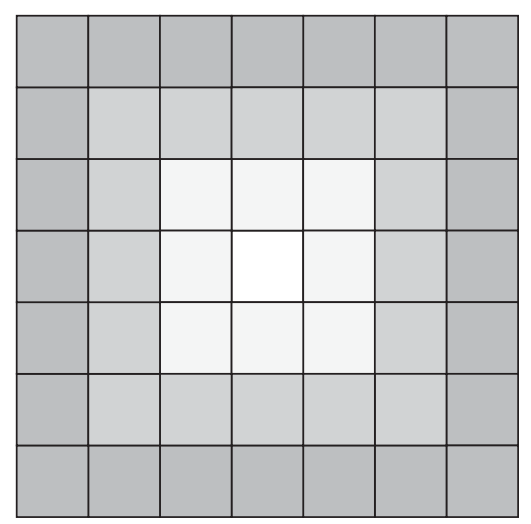

FIgURE 1: The sets $B_{1}, B_{2}, B_{3}$, and $B_{4}$, when $d=2$.

Since only configurations belonging to $H$ can result in cell $C_{N, \bar{j}}$ failing after exactly $n_{0}$ steps, the term on the second line of (3.1) equals

$$
\sum_{h \in H} q_{h} c_{h} b_{N, \bar{j}}(t) \frac{(\mathcal{K} t)^{n_{0}}}{n_{0} !} \mathrm{e}^{-K t}=q b_{N, \bar{j}}(t) \frac{(\mathcal{K} t)^{n_{0}}}{n_{0} !} \mathrm{e}^{-K t}
$$

where $b_{N, \bar{j}}(t)$ is the probability of no particle in $C_{N, \bar{j}}^{\mathrm{c}}$ reaching $C_{N, \bar{j}}$ prior to $t$.

When $N=1$ and, hence, $\bar{J}=\overline{1}$, there is only one cell, and it follows that $b_{1, \overline{1}}(t)=1$. In the case of the infinite system, $\mathrm{P}\left\{\tilde{\tau}_{\overline{1}} \leq t\right\}$ is determined as in (3.1), (3.2), and (3.3), the only computational difference being that $b_{N, \bar{j}}(t)$ is replaced with $b(t)$, where

$$
1 \geq b_{N, \bar{j}}(t) \geq b(t)
$$

We will show that $b(t) \rightarrow 1$ as $t \rightarrow 0$ in Lemma 3.1. To prove Lemma 3.1, we need the following inequality (see [10, p. 30]). Let $Y$ be a Poisson-distributed random variable of mean $\lambda$. Then, for $k=0,1, \ldots$,

$$
\mathrm{P}\{Y \geq k\} \leq\left(\frac{\lambda \mathrm{e}}{k}\right)^{k}
$$

Lemma 3.1. As $t \rightarrow 0, b(t) \rightarrow 1$.

Proof. We construct blocks of cells $B_{k}$, as follows. Let $B_{1}=C_{\overline{1}}$. There are $3^{d}-1$ cells, whose union we denote by $B_{2}$, bordering $B_{1}$. Each cell in $B_{2}$ contains $\mathcal{K}$ particles and each particle must make at least one jump in order to reach $B_{1}$. Suppose that we have constructed $B_{k}$, for some $k$. There are $(2 k+1)^{d}-(2 k-1)^{d}$ cells, whose union we denote by $B_{k+1}$, bordering $B_{k}$. Each cell in $B_{k}$ has $\mathcal{K}$ particles and each particle must make at least $(k-2) l+1$ moves in order to reach $B_{1}$. In Figure $1, B_{1}$ is the cell in the center, $B_{2}$ are the eight cells surrounding $B_{1}$, $B_{3}$ are the 16 cells surrounding $B_{2}$, and $B_{4}$ are the 24 cells surrounding $B_{3}$. This construction provides a method of bounding the length of time it takes particles in $B_{k}, k=2,3, \ldots$, to reach $B_{1}$. 
It follows that $b(t)$ is the probability that no particle in $B_{1}^{\mathrm{c}}$ reaches $B_{1}$ prior to $t$. Let $Y$ be a random variable having a Poisson distribution of mean $t$. We note that

$$
\begin{aligned}
1-b(t) & \leq \sum_{k=2}^{\infty} \mathcal{K}(2 k+1)^{d} \mathrm{P}\{Y \geq l(k-2)+1\} \\
& \leq \sum_{k=2}^{\infty} \mathcal{K}(2 k+1)^{d}\left(\frac{\mathrm{e} t}{l(k-2)+1}\right)^{l(k-2)+1}
\end{aligned}
$$

which converges for any $t$. The result now follows from the dominated convergence theorem.

We determine $u_{N}$ by solving

$$
\frac{\left(\mathcal{K} u_{N}\right)^{n_{0}}}{n_{0} !}=\frac{1}{N^{d}}
$$

Lemma 3.2. Set

$$
u_{N}=\frac{1}{\mathcal{K}}\left(\frac{n_{0} !}{N^{d}}\right)^{1 / n_{0}}
$$

Then

$$
\mathrm{P}\left\{\tau_{N, \bar{j}} \leq u_{N}\right\}=\frac{q}{N^{d}}+o\left(\frac{1}{N^{d}}\right) .
$$

Proof. Note that $u_{N} \rightarrow 0$ as $N \rightarrow \infty$. Hence, $\exp \left(-\mathcal{K} u_{N}\right)=1+o(1)$ and $b\left(u_{N}\right)=$ $1+o(1)$. In addition,

$$
N^{d} \frac{\left(\mathcal{K} u_{N}\right)^{n_{0}+1}}{\left(n_{0}+1\right) !}=C N^{d}\left(\frac{1}{N^{d}}\right)^{1+1 / n_{0}} \rightarrow 0
$$

as $N \rightarrow \infty$, where $C$ is a constant. It follows from (3.1), (3.2), and (3.3), applied to $\tau_{1, \overline{1}}$ and $\tilde{\tau}_{\overline{1}}$, that

$$
\mathrm{P}\left\{\tau_{1, \overline{1}} \leq u_{N}\right\}=\frac{q}{N^{d}}(1+o(1))+o\left(\frac{1}{N^{d}}\right)=\frac{q}{N^{d}}+o\left(\frac{1}{N^{d}}\right)
$$

and

$$
\mathrm{P}\left\{\tilde{\tau}_{\overline{1}} \leq u_{N}\right\}=(1+o(1)) \frac{q}{N^{d}}(1+o(1))+o\left(\frac{1}{N^{d}}\right)=\frac{q}{N^{d}}+o\left(\frac{1}{N^{d}}\right) .
$$

Since

$$
\mathrm{P}\left\{\tilde{\tau}_{\overline{1}} \leq u_{N}\right\} \leq \mathrm{P}\left\{\tau_{N, \bar{j}} \leq u_{N}\right\} \leq \mathrm{P}\left\{\tau_{1, \overline{1}} \leq u_{N}\right\}
$$

the result follows.

Lemma 3.3. For any $a>0$,

$$
\lim _{N \rightarrow \infty} \frac{\mathrm{P}\left\{\tau_{N, \bar{j}} \leq a u_{N}\right\}}{\mathrm{P}\left\{\tau_{N, \bar{j}} \leq u_{N}\right\}}=a^{n_{0}} .
$$

Proof. From (3.1), (3.2), (3.3), and Lemma 3.1,

$$
\frac{\mathrm{P}\left\{\tau_{N, \bar{j}} \leq t\right\}}{t^{n_{0}}} \rightarrow C
$$

as $t \rightarrow 0$, for some constant $C$. Since $u_{N} \rightarrow 0$ as $N \rightarrow \infty$, the result follows. 


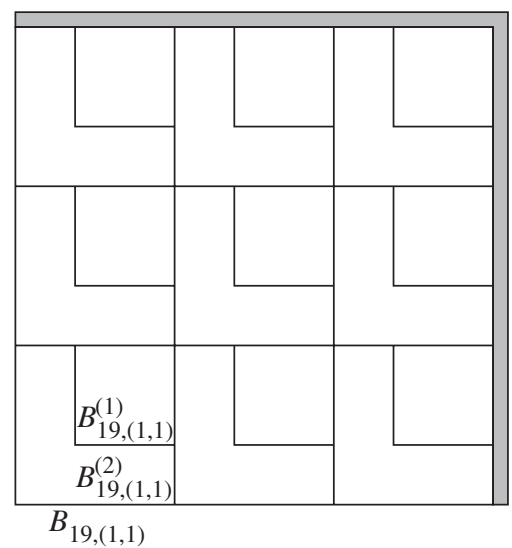

FIGURE 2: The sets $B_{19,\left(i_{1}, i_{2}\right)}, B_{19,\left(i_{1}, i_{2}\right)}^{(1)}$, and $B_{19,\left(i_{1}, i_{2}\right)}^{(2)}$. Those with $i_{1}=i_{2}=1$ are explicitly labeled.

We next need to develop a mixing condition analogous to the condition $D\left(u_{N}\right)$ given in [5]. To this end, choose a positive integer $k$ and define $N^{\prime}=\lfloor N / k\rfloor$, the greatest integer less than or equal to $N / k$. For $\bar{\imath}=\left(i_{1}, \ldots, i_{d}\right), i_{j}=1, \ldots, k$, and $m$ such that $k<m<N^{\prime}$, let

$$
\begin{aligned}
B_{N, \bar{l}} & =\prod_{j=1}^{d}\left\{N^{\prime}\left(i_{j}-1\right) l+1, \ldots, N^{\prime} i_{j} l\right\}, \\
B_{N, \bar{l}}^{(1)} & =\prod_{j=1}^{d}\left\{\left(N^{\prime}\left(i_{j}-1\right)+m\right) l+1, \ldots, N^{\prime} i_{j} l\right\}, \\
B_{N, \bar{l}}^{(2)} & =B_{N, \bar{l}} / B_{N, \bar{l}}^{(1)} .
\end{aligned}
$$

These sets are described in Figure 2, where $d=2, N=19, k=3$, and $m=4$, meaning that $N^{\prime}=6$. There, $B_{19,(1,1)}$ is the block in the lower left-hand corner. It is divided into two parts: the first part, $B_{19,(1,1)}^{(1)}$, is in the upper right-hand corner of $B_{19,(1,1)}$, and $B_{19,(1,1)}^{(2)}$ is the remainder of $B_{19,(1,1)}$. Notice that the union of the blocks is not necessarily the whole set. The uncovered part of the set is represented by the shaded region in the figure. To account for this region, we introduce the following blocks.

Let $\bar{l}=\left(i_{1}, \ldots, i_{d}\right)$, with $i_{j}=1, \ldots, k+1$, such that at least one component satisfies $i_{j}=k+1$. Let $A=\left\{j: i_{j}=k+1\right\}$ and let

$$
\begin{aligned}
& B_{N, \bar{i}}=\prod_{j \in A}\left\{\left(N-N^{\prime}\right) l+1, \ldots, N l\right\} \prod_{j \in A^{\mathrm{c}}}\left\{N^{\prime}\left(i_{j}-1\right) l+1, \ldots, N^{\prime} i_{j} l\right\}, \\
& B_{N, \bar{i}}^{(1)}=\prod_{j \in A}\left\{\left(N-N^{\prime}\right) l+1, \ldots, k N^{\prime} l\right\} \prod_{j \in A^{\mathrm{c}}}\left\{\left(N^{\prime}\left(i_{j}-1\right)+m\right) l+1, \ldots, N^{\prime} i_{j} l\right\}, \\
& B_{N, \bar{i}}^{(2)}=B_{N, \bar{l}} / B_{N, \bar{i}}^{(1)} .
\end{aligned}
$$

In Figure 3 , the blocks $B_{19,(1,4)}, B_{19,(4,1)}$, and $B_{19,(4,4)}$ are the same size as $B_{19,(1,1)}$, the blocks $B_{19,(1,4)}^{(1)}, B_{19,(4,1)}^{(1)}$, and $B_{19,(4,4)}^{(1)}$ are larger than $B_{19,(1,1)}^{(1)}$, and the blocks $B_{19,(1,4)}^{(2)}, B_{19,(4,1)}^{(2)}$, and 


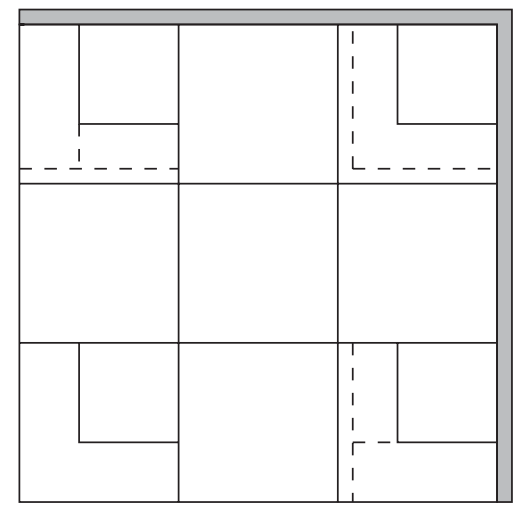

FIGURE 3: The sets $B_{19,\left(i_{1}, i_{2}\right)}, B_{19,\left(i_{1}, i_{2}\right)}^{(1)}$, and $B_{19,\left(i_{1}, i_{2}\right)}^{(2)}$, when at least one component satisfies $i_{j}=4$.

$B_{19,(4,4)}^{(2)}$ are smaller than $B_{19,(1,1)}^{(2)}$. Not shown are the blocks $B_{19,(4,2)}, B_{19,(4,3)}, B_{19,(2,4)}$, and $B_{19,(3,4)}$, but similar remarks hold for these blocks.

Associate with an $\bar{l}$ such that $i_{j}=k+1$ for some $j$, an $\bar{l}^{\prime}$ such that

$$
i_{j}^{\prime}= \begin{cases}1 & \text { if } i_{j}=k+1 \\ i_{j} & \text { otherwise }\end{cases}
$$

Consider a block $B_{N, \bar{l}^{\prime}}$ defined as in (3.4). By the symmetry of $S_{N}$, we have

$$
\mathrm{P}\left\{\tau_{B_{N, \bar{l}}^{(2)}} \leq u_{N}<\tau_{B_{N, \bar{l}}^{(1)}}\right\} \leq \mathrm{P}\left\{\tau_{B_{N, \bar{i}^{\prime}}^{(2)}} \leq u_{N}<\tau_{B_{N, \bar{l}^{\prime}}^{(1)}}\right\} .
$$

As shown in Figure 4, by flipping the boxes $B_{19,(4,1)}, B_{19,(1,4)}$, and $B_{19,(4,4)}$ we can compare the lifetimes of their cells to the lifetimes of the corresponding cells in $B_{19,(1,1)}$. Similar comments apply to the other boxes, when one of the components satisfies $i_{j}=4$.

Lemma 3.4. Let $A$ be a collection of cells in $S_{N}$, and let $p$ be the probability that at least one particle more than $\left\lfloor\frac{1}{2} m\right\rfloor$ cells removed from A reaches A prior to $u_{N}$. Then there is a constant $K_{1}$, independent of $N$, such that

$$
p \leq \mathcal{K} N^{d} \mathrm{e}^{3 K_{1}} \mathrm{e}^{-l\lfloor m / 2\rfloor}
$$

Proof. In order for a particle more than $\left\lfloor\frac{1}{2} m\right\rfloor$ cells removed from $A$ to reach $A$ in $u_{N}$ units of time, it must make at least $l\left\lfloor\frac{1}{2} m\right\rfloor$ jumps in $u_{N}$ time units. To bound the probability of the particle making at least $l\left\lfloor\frac{1}{2} m\right\rfloor$ jumps prior to $u_{N}$, let $X$ be a random variable with a Poisson distribution of mean $u_{N}$, and set $Y=X-l\left\lfloor\frac{1}{2} m\right\rfloor$. Then, the probability that a particle makes at least $l\left\lfloor\frac{1}{2} m\right\rfloor$ jumps prior to $u_{N}$ is

$$
\begin{aligned}
\mathrm{P}\left\{X \geq l\left\lfloor\frac{1}{2} m\right\rfloor\right\} & =\mathrm{P}\{Y \geq 0\} \\
& \leq \mathrm{E}\left[\mathrm{e}^{\theta Y}\right] \\
& =\mathrm{e}^{-\theta l\lfloor m / 2\rfloor} \exp \left(u_{N}\left(\mathrm{e}^{\theta}-1\right)\right),
\end{aligned}
$$


(a)

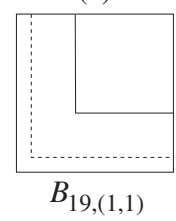

(b)

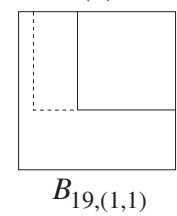

(c)

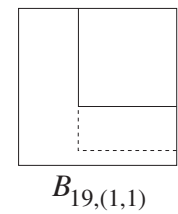

FIGURE 4: Comparing the sets $B_{19,\left(i_{1}, i_{2}\right)}, B_{19,\left(i_{1}, i_{2}\right)}^{(1)}$, and $B_{19,\left(i_{1}, i_{2}\right)}^{(2)}$ to $B_{19,(1,1)}$ when at least one component satisfies $i_{j}=4$. In (a) we compare $B_{19,(1,1)}$ to $B_{19,(4,4)}$, in (b) we compare $B_{19,(1,1)}$ to $B_{19,(4,1)}$, and in (c) we compare $B_{19,(1,1)}$ to $B_{19,(1,4)}$.

where $\theta \geq 0$. Since $u_{N} \rightarrow 0$ as $N \rightarrow \infty$, there is a $K_{1}$ such that

$$
\sup _{N} u_{N} \leq K_{1}
$$

Setting $\theta=1$ gives

$$
\mathrm{P}\left\{X \geq l\left\lfloor\frac{1}{2} m\right\rfloor\right\} \leq \mathrm{e}^{-l\lfloor m / 2\rfloor} \mathrm{e}^{3 K_{1}} .
$$

Since the set consists of $\mathcal{K} N^{d}$ particles, we have

$$
p \leq \mathcal{K} N^{d} \mathrm{e}^{3 K_{1}} \mathrm{e}^{-l\lfloor m / 2\rfloor}
$$

and the proof is complete.

Lemma 3.5. For $i=1,2$, let $A_{i}$ be a collection of cells in $S_{N}$. Assume that lm is the minimum number of jumps required for a particle in $A_{1}$ to reach $A_{2}$. Let $\tau_{A_{i}}$ be the minimum of the lifetimes of the cells in $A_{i}$. Then

$$
\left|\mathrm{P}\left\{\tau_{A_{1}} \leq u_{N}, \tau_{A_{2}} \leq u_{N}\right\}-\mathrm{P}\left\{\tau_{A_{1}} \leq u_{N}\right\} \mathrm{P}\left\{\tau_{A_{2}} \leq u_{N}\right\}\right| \leq 8 \mathcal{K} N^{d} \mathrm{e}^{3 K_{1}} \mathrm{e}^{-l\lfloor m / 2\rfloor} .
$$

In particular, if $B_{N, \bar{l}_{1}}^{(1)}$ and $B_{N, \bar{l}_{2}}^{(1)}$ are defined as in (3.4), with $\bar{\imath}_{1} \neq \bar{\imath}_{2}$, then

$$
\left|\mathrm{P}\left\{\tau_{B_{N, \bar{l}_{1}}^{(1)}} \leq u_{N}, \tau_{B_{N, \bar{l}_{2}}^{(1)}} \leq u_{N}\right\}-\mathrm{P}\left\{\tau_{B_{N, \bar{l}_{1}}^{(1)}} \leq u_{N}\right\} \mathrm{P}\left\{\tau_{B_{N, \bar{l}_{2}}^{(1)}} \leq u_{N}\right\}\right| \leq 8 \mathcal{K} N^{d} \mathrm{e}^{3 K_{1}} \mathrm{e}^{-l\lfloor m / 2\rfloor}
$$

Proof. For $h=1,2$, let $\tilde{A}_{i}$ be the union of $A_{i}$ and all locations that are within $l\left\lfloor\frac{1}{2} m\right\rfloor$ jumps of $A_{i}$. Let $\mathcal{A}_{i}$ be the event that no particle starting in $\tilde{A}_{i}^{\mathrm{c}}$ reaches $A_{i}$ prior to $u_{N}$. Color white the particles initially in $\tilde{A}_{1}$, color orange those initially in $\tilde{A}_{2}$ and color the remaining particles purple. Let $\tilde{\tau}_{A_{1}}$ and $\tilde{\tau}_{A_{2}}$ be the lengths of time for the number of white particles in one of the cells of $A_{1}$, and, respectively, the number of orange particles in one of the cells of $A_{2}$, to fall below $\mathcal{K}-\kappa$. Then $\tilde{\tau}_{A_{1}}$ and $\tilde{\tau}_{A_{2}}$ are independent. Conditioned on $\mathcal{A}_{1} \cap \mathcal{A}_{2}$, the events $\left\{\tilde{\tau}_{A_{1}} \leq u_{N}, \tilde{\tau}_{A_{2}} \leq u_{N}\right\}$ and $\left\{\tau_{A_{1}} \leq u_{N}, \tau_{A_{2}} \leq u_{N}\right\}$ have the same probability. Letting

$$
c=2 \mathcal{K} N^{d} \mathrm{e}^{3 K_{1}} \mathrm{e}^{-l\lfloor m / 2\rfloor},
$$


we have

$$
\begin{aligned}
\mid \mathrm{P}\left\{\tau_{A_{1}} \leq\right. & \left.u_{N}, \tau_{A_{2}} \leq u_{N}\right\}-\mathrm{P}\left\{\tau_{A_{1}} \leq u_{N}\right\} \mathrm{P}\left\{\tau_{A_{2}} \leq u_{N}\right\} \mid \\
= & \mid \mathrm{P}\left\{\tau_{A_{1}} \leq u_{N}, \tau_{A_{2}} \leq u_{N} \mid \mathcal{A}_{1} \cap \mathcal{A}_{2}\right\} \mathrm{P}\left(\mathcal{A}_{1} \cap \mathcal{A}_{2}\right) \\
& +\mathrm{P}\left\{\tau_{A_{1}} \leq u_{N}, \tau_{A_{2}} \leq u_{N},\left(\mathcal{A}_{1} \cap \mathcal{A}_{2}\right)^{\mathrm{c}}\right\} \\
& -\mathrm{P}\left\{\tau_{A_{1}} \leq u_{N}\right\} \mathrm{P}\left\{\tau_{A_{2}} \leq u_{N}\right\}\left(\mathrm{P}\left(\mathcal{A}_{1} \cap \mathcal{A}_{2}\right)+\mathrm{P}\left(\left(\mathcal{A}_{1} \cap \mathcal{A}_{2}\right)^{\mathrm{c}}\right)\right) \mid \\
\leq & \left|\mathrm{P}\left\{\tau_{A_{1}} \leq u_{N}, \tau_{A_{2}} \leq u_{N} \mid \mathcal{A}_{1} \cap \mathcal{A}_{2}\right\}-\mathrm{P}\left\{\tau_{A_{1}} \leq u_{N}\right\} \mathrm{P}\left\{\tau_{A_{2}} \leq u_{N}\right\}\right| \mathrm{P}\left(\mathcal{A}_{1} \cap \mathcal{A}_{2}\right) \\
& +\mathrm{P}\left\{\tau_{A_{1}} \leq u_{N}, \tau_{A_{2}} \leq u_{N},\left(\mathcal{A}_{1} \cap \mathcal{A}_{2}\right)^{\mathrm{c}}\right\} \\
& +\mathrm{P}\left\{\tau_{A_{1}} \leq u_{N}\right\} \mathrm{P}\left\{\tau_{A_{2}} \leq u_{N}\right\} \mathrm{P}\left(\left(\mathcal{A}_{1} \cap \mathcal{A}_{2}\right)^{\mathrm{c}}\right) \\
\leq & \left|\mathrm{P}\left\{\tilde{\tau}_{A_{1}} \leq u_{N}\right\} \mathrm{P}\left\{\tilde{\tau}_{A_{2}} \leq u_{N}\right\}-\mathrm{P}\left\{\tau_{A_{1}} \leq u_{N}\right\} \mathrm{P}\left\{\tau_{A_{2}} \leq u_{N}\right\}\right|+2 c .
\end{aligned}
$$

Furthermore,

$$
\left|\mathrm{P}\left\{\tilde{\tau}_{A_{i}} \leq u_{N}\right\}-\mathrm{P}\left\{\tau_{A_{i}} \leq u_{N}\right\}\right| \leq\left|\mathrm{P}\left\{\tilde{\tau}_{A_{i}} \leq u_{N}\right\}-\mathrm{P}\left\{\tau_{A_{i}} \leq u_{N} \mid \mathcal{A}_{i}\right\}\right| \mathrm{P}\left(\mathcal{A}_{i}\right)+2 \mathrm{P}\left(\mathcal{A}_{i}^{\mathrm{c}}\right) \leq c .
$$

Since

$$
\begin{aligned}
& \left|\mathrm{P}\left\{\tilde{\tau}_{A_{1}} \leq u_{N}\right\} \mathrm{P}\left\{\tilde{\tau}_{A_{2}} \leq u_{N}\right\}-\mathrm{P}\left\{\tau_{A_{1}} \leq u_{N}\right\} \mathrm{P}\left\{\tau_{A_{2}} \leq u_{N}\right\}\right| \\
& \quad \leq\left|\mathrm{P}\left\{\tilde{\tau}_{A_{1}} \leq u_{N}\right\}-\mathrm{P}\left\{\tau_{A_{1}} \leq u_{N}\right\}\right|+\left|\mathrm{P}\left\{\tilde{\tau}_{A_{2}} \leq u_{N}\right\}-\mathrm{P}\left\{\tau_{A_{2}} \leq u_{N}\right\}\right|,
\end{aligned}
$$

the result follows from first inserting (3.6) into (3.7) and then inserting (3.7) into (3.5).

Let $c_{N, m}=8 \mathcal{K} N^{d} \mathrm{e}^{3 K_{1}} \mathrm{e}^{-l\lfloor m / 2\rfloor}$, which was used in the proof of Lemma 3.5. The constants $c_{N, m}$ play the same role as the constants $\alpha_{n, m}$ given in [5].

Lemma 3.6. Let $C_{N}=\left\{\bar{l}: 0 \leq i_{j} \leq k\right\}$ and $D_{N}=\left\{\bar{l}: 0 \leq i_{j} \leq k+1\right\}$. Then

$$
\begin{aligned}
\mid \mathrm{P}\left\{\tau_{N}\right. & \left.>u_{N}\right\}-\prod_{\bar{i} \in C_{N}} \mathrm{P}\left\{\tau_{B_{N, \bar{i}}}>u_{N}\right\} \mid \\
\leq & \left(\sum_{\bar{i} \in C_{N}}+\sum_{\bar{i} \in D_{N}}\right) \mathrm{P}\left\{\tau_{B_{N, \bar{l}}^{(1)}}>u_{N}>\tau_{B_{N, \bar{l}}^{(2)}}\right\}+\left(k^{d}-1\right) c_{N, m} .
\end{aligned}
$$

Proof. The inequalities (3.9), (3.10), and (3.11), i.e.

$$
\begin{gathered}
0 \leq \mathrm{P}\left(\bigcap_{\bar{i} \in C_{N}}\left\{\tau_{B_{N, \bar{i}}^{(1)}}>u_{N}\right\}\right)-\mathrm{P}\left\{\tau_{N}>u_{N}\right\} \\
\leq \sum_{\bar{i} \in D_{N}} \mathrm{P}\left\{\tau_{B_{N, \bar{i}}^{(1)}}>u_{N} \geq \tau_{B_{N, \bar{i}}^{(2)}}\right\} \\
\left|\mathrm{P}\left(\bigcap_{\bar{i} \in C_{N}}\left\{\tau_{B_{N, \bar{l}}^{(1)}}>u_{N}\right\}\right)-\prod_{\bar{i} \in C_{N}} \mathrm{P}\left\{\tau_{B_{N, \bar{l}}^{(1)}}>u_{N}\right\}\right| \leq\left(k^{d}-1\right) c_{N, m},
\end{gathered}
$$

and

$$
\left|\prod_{\bar{\imath} \in C_{N}} \mathrm{P}\left\{\tau_{B_{N, \bar{l}}^{(1)}}>u_{N}\right\}-\prod_{\bar{\imath} \in C_{N}} \mathrm{P}\left\{\tau_{B_{N, \bar{l}}}>u_{N}\right\}\right| \leq \sum_{\bar{\imath} \in C_{N}} \mathrm{P}\left\{\tau_{B_{N, \bar{l}}^{(1)}}>u_{N} \geq \tau_{B_{N, \bar{l}}^{(2)}}\right\},
$$

are derived in a manner similar to the inequalities (i), (ii), and (iii) given in Lemma 3.3.1 of [5]. Adding (3.9), (3.10), and (3.11) gives (3.8), and completes the proof. 
Lemma 3.7. Let $m=N^{1 / 2}$. Then

$$
\sup _{\bar{i} \in D_{N}} \mathrm{P}\left\{\tau_{B_{N, \bar{l}}^{(1)}}>u_{N} \geq \tau_{B_{N, \bar{l}}^{(2)}}\right\} \rightarrow 0
$$

as $N \rightarrow \infty$.

Proof. Since $\tau_{N, \bar{j}}$ is stochastically larger than $\tau_{1, \overline{1}}$,

$$
\sup _{\bar{j}} \mathrm{P}\left\{\tau_{N, \bar{j}} \leq u_{N}\right\} \leq \mathrm{P}\left\{\tau_{1, \overline{1}} \leq u_{N}\right\} \leq \frac{q}{N^{d}}+o\left(\frac{1}{N^{d}}\right) .
$$

Then, since there are at most $m^{d}$ cells in each $B_{N, \bar{l}}^{(2)}$,

$$
\sup _{\bar{i} \in D_{N}} \mathrm{P}\left\{\tau_{B_{N, \bar{i}}^{(1)}}>u_{N} \geq \tau_{B_{N, \bar{\imath}}^{(2)}}\right\} \leq N^{d / 2} \sup _{\bar{j}} \mathrm{P}\left\{\tau_{N, \bar{j}} \leq u_{N}\right\} \rightarrow 0
$$

as $N \rightarrow \infty$, and the proof is complete.

Lemma 3.8. With $m=N^{1 / 2}$,

$$
\left|\mathrm{P}\left\{\tau_{N}>u_{N}\right\}-\prod_{\bar{\imath} \in C_{N}} \mathrm{P}\left\{\tau_{B_{N, \bar{i}}}>u_{N}\right\}\right| \rightarrow 0
$$

as $N \rightarrow \infty$.

Proof. Note that $C_{N} \subset D_{N}$ and that $D_{N}$ has $(k+1)^{d}$ members. Moreover, $c_{N, N^{1 / 2}} \rightarrow 0$ as $N \rightarrow \infty$. The result now follows from Lemmas 3.6 and 3.7.

We now give a condition analogous to the condition $D^{\prime}\left(u_{N}\right)$ of [5]. Recall that the sets $C_{N}$ and $B_{N, \bar{l}}$ depend on $k$.

Lemma 3.9. As $k \rightarrow \infty$,

$$
\lim \sup _{N} N^{d} \sup _{\bar{l} \in C_{N}} \sup _{\bar{j} \in B_{N, \bar{l}}} \sum_{\bar{j}^{\prime} \in B_{N, \bar{l}} /\{\bar{j}\}} \mathrm{P}\left\{\tau_{N, \bar{j}} \leq u_{N}, \tau_{N, \bar{j}^{\prime}} \leq u_{N}\right\} \rightarrow 0 .
$$

Proof. Let $C_{N, \bar{j}_{1}}$ and $C_{N, \bar{j}_{2}}$ be distinct cells in $S_{N}$. Color orange the particles initially in $C_{N, \bar{j}_{1}}$, color white those initially in $C_{N, \bar{j}_{2}}$, and color the remainder of the particles purple: once a particle leaves either $C_{N, \bar{j}_{1}}$ or $C_{N, \bar{j}_{2}}$, it turns purple. Let $\tau^{(1)}$ denote the time required for $\kappa$ orange particles to leave $C_{N, \bar{j}_{1}}$ and let $\tau^{(2)}$ denote the time required for $\kappa$ white particles to leave $C_{N, \bar{j}_{2}}$. It is clear that $\tau^{(1)}$ and $\tau^{(2)}$ are independent random variables, that they have the same distribution as $\tau_{1, \overline{1}}$, and that, if both $\tau_{N, \bar{j}_{1}}$ and $\tau_{N, \bar{j}_{2}}$ are less than or equal to $u_{N}$, so are both $\tau^{(1)}$ and $\tau^{(2)}$. Thus, by Lemma 3.2,

$$
\begin{aligned}
\mathrm{P}\left\{\tau_{N, \bar{j}_{1}} \leq u_{N}, \tau_{N, \bar{j}_{2}} \leq u_{N}\right\} & \leq \mathrm{P}\left\{\tau^{(1)} \leq u_{N}, \tau^{(2)} \leq u_{N}\right\} \\
& =\left(\frac{q}{N^{d}}+o\left(\frac{1}{N^{d}}\right)\right)^{2} \\
& =\frac{q^{2}}{N^{2 d}}+o\left(\frac{1}{N^{2 d}}\right) .
\end{aligned}
$$


Since $B_{N, \bar{l}}$ has at most $(N / k)^{d}$ cells,

$$
\sup _{\bar{\imath} \in C_{N}} \sup _{j \in B_{N, \bar{l}}} \sum_{\bar{j}^{\prime} \in B_{N, \bar{l}} /\{\bar{j}\}} \mathrm{P}\left\{\tau_{N, \bar{j}} \leq u_{N}, \tau_{N, \bar{j}^{\prime}} \leq u_{N}\right\} \leq \frac{q^{2}}{(k N)^{d}}+o\left(\frac{1}{N^{d}}\right) .
$$

By multiplying the above equation by $N^{d}$, taking the limit supremum over $N$, and letting $k \rightarrow \infty$, we obtain the result.

The next result is an application to our problem of Theorem 3.4.1 of [5]. We include the proof so that the reader does not have to translate notation between the two settings.

Lemma 3.10. As $N \rightarrow \infty$, we have $\mathrm{P}\left\{\tau_{N}>u_{N}\right\} \rightarrow \mathrm{e}^{-q}$.

Proof. Fix an integer $k$. For each block $B_{N, \bar{l}}$,

$$
\left\{\tau_{B_{N, \bar{i}}} \leq u_{N}\right\}=\bigcup_{C_{N, \bar{j}} \in B_{N, \bar{l}}}\left\{\tau_{N, \bar{j}} \leq u_{N}\right\}
$$

Thus,

$$
\begin{gathered}
1-\sum_{C_{N, \bar{j}} \in B_{N, \bar{i}}} \mathrm{P}\left\{\tau_{N, \bar{j}} \leq u_{N}\right\}+\sum_{C_{N, \overline{j_{1}}} \neq C_{N, \bar{j}_{2}} \in B_{N, \bar{i}}} \mathrm{P}\left\{\tau_{N, \bar{j}_{1}} \leq u_{N}, \tau_{N, \bar{j}_{2}} \leq u_{N}\right\} \\
\quad \geq \mathrm{P}\left\{\tau_{B_{N, \bar{i}}} \leq u_{N}\right\} \geq 1-\sum_{C_{N, \bar{j}} \in B_{N, \bar{i}}} \mathrm{P}\left\{\tau_{N, \bar{j}} \leq u_{N}\right\}
\end{gathered}
$$

The first sum has $\lfloor N / k\rfloor^{d}$ terms and, so, converges to $q / k^{d}$ as $N \rightarrow \infty$, by Lemma 3.2. The second sum is bounded above by

$$
\lim \sup _{N}\left(\frac{N}{k}\right)^{d} \sup _{\bar{\imath} \in C_{N}} \sup _{\bar{j} \in B_{N, \bar{i}}} \sum_{\bar{J}^{\prime} \in B_{N, \bar{l}} /\{\bar{j}\}} \mathrm{P}\left\{\tau_{N, \bar{j}} \leq u_{N}, \tau_{N, \bar{j}^{\prime}} \leq u_{N}\right\}=o\left(\frac{1}{k^{d}}\right),
$$

by Lemma 3.9. Hence,

$$
1-\frac{q}{k^{d}}+o\left(\frac{1}{k^{d}}\right) \geq \lim \sup _{N} \mathrm{P}\left\{\tau_{B_{N, \bar{l}}} \leq u_{N}\right\} \geq \lim _{N} \mathrm{P}\left\{\tau_{B_{N, \bar{l}}} \leq u_{N}\right\} \geq 1-\frac{q}{k^{d}} .
$$

Taking the product over the $k^{d}$ blocks $B_{N, i}$ in $C_{N}$ gives, by Lemma 3.8,

$$
\left(1-\frac{q}{k^{d}}+o\left(\frac{1}{k^{d}}\right)\right)^{k^{d}} \geq \lim \sup _{N} \mathrm{P}\left\{\tau_{N} \leq u_{N}\right\} \geq \lim _{N} \inf _{N} \mathrm{P}\left\{\tau_{N} \leq u_{N}\right\} \geq\left(1-\frac{q}{k^{d}}\right)^{k^{d}} .
$$

The result now follows by letting $k \rightarrow \infty$.

Proof of Theorem 2.1. It follows from Lemmas 3.2 and 3.3 that

$$
\sum_{\bar{j} \in N^{d}} \mathrm{P}\left\{\tau_{N, \bar{j}} \leq x u_{N}\right\} \rightarrow q x^{n_{0}}
$$

as $N \rightarrow \infty$. Replacing $u_{N}$ by $x u_{N}$ and $q$ by $q x^{n_{0}}$ in Lemmas 3.4 to 3.10 gives

$$
\mathrm{P}\left\{\tau_{N}>x u_{N}\right\} \rightarrow \mathrm{e}^{-q x^{n} 0},
$$

and the proof is complete. 


\section{References}

[1] Barlow, R. E. and Proschan, F. (1981). Statistical Theory of Reliability and Life Testing. To Begin With, Silver Spring, MD.

[2] Bingham, N. H., Goldie, C. M. and Teugels, J. L. (1987). Regular Variation. Cambridge University Press.

[3] Griebel, M., JaGer, L. AND Voigt, A. (2004). Computing diffusion coefficients of intrinsic point defects in crystalline silicon. In Proc. 10th Internat. Conf. Composites Eng., ed. D. Hui, International Community for Composites Engineering, New Orleans, pp. 487-488.

[4] Gumbel, E. J. (1958). Statistics of Extremes. Columbia University Press, New York.

[5] Leadbetter, M. R., Lindgren, G. And Rootzén, H. (1983). Extremes and Related Properties of Random Sequences and Processes. Springer, New York.

[6] Lawless, J. F. (1982). Statistical Models and Methods for Lifetime Data. John Wiley, New York.

[7] Meeker, W. O. and Escobar, L. A. (1998). Statistical Methods for Reliability Data. John Wiley, New York.

[8] Neudeck, P. G. AND Powell, J. A. (2004). Performance limiting micropipe defects in silicon carbide wafers. Res. Rept., NASA Lewis Research Center. Available at http://www.grc.nasa.gov/WWW/SiC/publications/ micropipeEDLPaper.html.

[9] Resnick, S. I. (1987). Extreme Values, Regular Variation, and Point Processes. Springer, New York.

[10] RÉvész, P. (1994). Random Walks on Infinitely Many Particles. World Scientific, River Edge, NJ. 